\title{
Análise descritiva das dimensóes do burnout: um estudo com jovens trabalhadores
}

\author{
Marina Batista Chaves Azevedo de Souza ${ }^{\mathrm{a}, \mathrm{b}}$ (D), Diogo Henrique Helal ${ }^{\mathrm{c} d}$ (i), \\ Kely César Martins de Paiva ${ }^{\mathrm{e}}$ (D) \\ aDepartamento de Terapia Ocupacional, Universidade Federal de Sergipe - UFS, Lagarto, SE, Brasil

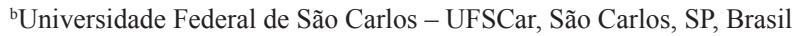 \\ ${ }^{\mathrm{c}}$ Fundação Joaquim Nabuco - FUNDAJ, Ministério da Educação - MEC, Recife, PE, Brasil. \\ ${ }^{\mathrm{d} C e n t r o ~ U n i v e r s i t a ́ r i o ~ F B V ~-~ U N I F B V, ~ R e c i f e, ~ P E, ~ B r a s i l . ~}$ \\ eUniversidade Federal de Minas Gerais - UFMG, Belo Horizonte, MG, Brasil.
}

\begin{abstract}
Resumo: Introdução: A literatura indica que jovens são mais suscetíveis a vivenciarem pressão e estresse no trabalho e apontam que essa população é mais inexperiente, tem dificuldade de controlar sua atividade laboral e de desenvolver papéis de agente. Assim, é importante pensar sobre o trabalho de jovens e os sofrimentos mentais que podem acarretar em patologias ou transtornos comportamentais do trabalho. Objetivo: Investigar o burnout analisando como estão configuradas a realização profissional, exaustão emocional e despersonalização no trabalho de jovens trabalhadores. Método: A pesquisa é exploratória, descritiva, de campo, quantitativa e com utilização de análise estatística descritiva uni e bivariada para analisar as respostas obtidas através do Inventário Maslach Burnout (MBI). Resultados: Através do MBI, aponta-se que os jovens apresentam altos níveis de realização profissional, o que parece incentivar índices de baixo a médio nas dimensões da exaustão emocional e despersonalização. Embora o burnout não tenha sido identificado, os comportamentos de risco experimentados por jovens são notáveis através dos valores máximos encontrados nas dimensões do inventário. Conclusão: A pesquisa não identificou resultados semelhantes com estudos atuais sobre burnout e jovens, indicando a necessidade de propor métodos de mensuração quantitativos mais específicos e pesquisas com abordagens qualitativas para melhor compreensão das idiossincrasias de jovens trabalhadores brasileiros. A existência de jovens que responderam valores altos para exaustão emocional ou despersonalização, indica que é necessária a reflexão sobre a individualidade desses trabalhadores, assim como os fatores subjetivos do trabalho que influenciam diretamente na vida do jovem e no seu desenvolvimento nas atividades das empresas.
\end{abstract}

Palavras-chave: Burnout, Saúde do Trabalhador, Juventude, Saúde Mental.

\section{Descriptive analysis of burnout dimensions: a study about the work of young learning}

\begin{abstract}
Introduction: The literature indicates that young workers would be more susceptible to situations of pressure or stress that shake the human psyche, since this public is indicated as being more prone to oppressive and violent circumstances at work. Thus, the onset of suffering from work is frequent, leading to work-related pathologies or behavioral disorders related to work. Objective: To describe the dimensions of burnout syndrome discussed by the Maslach model in the context of young workers. Method: Characterized as exploratory, descriptive, field, quantitative, and using uni and bivariate descriptive statistical analysis to evaluate the responses obtained through the Maslach Burnout Inventory (MBI). Results: Through the MBI, it is pointed out that young people
\end{abstract}

Autor para correspondência: Marina Batista Chaves Azevedo de Souza, Universidade Federal de São Carlos, Rodovia Washington Luis, Km 235, CEP 13565-905, São Carlos, SP, Brasil, e-mail: marinabs91@hotmail.com

Recebido em Set. 2, 2018; $1^{\text {a }}$ Revisão em Nov. 23, 2018; 2a Revisão em Maio 10, 2019; Aceito em Jul. $20,2019$. 
present high levels of professional achievement, which seems to encourage them to have low to medium indices also in the dimensions of emotional exhaustion and depersonalization. Yet, although burnout syndrome itself has not been found, risky and propensity behaviors experienced by young people are remarkable. Conclusion: The present research did not identify similar results with current and more accessible studies on the subjects, which indicates the need for new research on the subject, in order to identify peculiar characteristics of the study population and to propose methods of measurement more specific and indicated to this context. The results indicate the need for intervention in companies in order to allow greater enrichment of the activities developed by young people, avoiding the emergence of situations that may lead to suffering at work.

Keywords: Burnout, Occupational Health, Young Adult, Mental Health.

\section{Introdução}

Diversas são as possibilidades e motivos relacionados à entrada e à permanência de jovens no mercado de trabalho. A busca pelo sustento de uma família, por maior autonomia, a vontade de pertencer ao mercado de consumo ou o estabelecimento de uma renda própria, podem ser considerados, por exemplo, como potenciais motivos de escolha por adentrar neste mercado (GUERREIRO; ABRANTES, 2005). Há, entretanto, questóes acerca dos jovens trabalhadores e da sua inserção e permanência no mercado de trabalho que demandam maior aprofundamento pela literatura.

De acordo com Paiva et al. (2013), uma baixa experiência profissional e permanências mais longas nos sistemas educacionais fazem com que os jovens tenham maior dificuldade na inserção no mercado de trabalho. Essa questão é agravada quando se trata de empregos com a remuneração acima da média. Destaque-se, ainda, que são comuns aos jovens maior rotatividade nas empresas, o que leva a um menor tempo para apropriação do trabalho e estabelecimento de redes de relacionamento no trabalho, por exemplo.

Para Guerreiro e Abrantes (2005), a entrada de jovens no mercado de trabalho é por si só uma realidade multifacetada. São analisados pela literatura, diversos motivos e possibilidades de trajetórias entre a juventude e a idade adulta, que variam em função da classe social e do gênero, podendo se constituir em uma trajetória menos ou mais desfavorecida. A depender da trajetória, os jovens iráo experienciar uma entrada mais precoce no mercado de trabalho, ou se dedicarão mais aos estudos, ingressando mais tardiamente nas atividades laborais.

No Brasil, o estatuto da juventude considera jovens os indivíduos entre 15 e 29 anos. Há, contudo, alguns programas, como o Jovens Aprendizes, que traz uma faixa etária distinta para a definição de juventude: 14 a 24 anos. Isso indica que há diversos pontos de vista na literatura e na legislação acerca da faixa etária e das características dessa população.
Sobre os jovens brasileiros e sua realidade laboral, cumpre destacar que o último Censo indicou que a média de desocupação dos jovens com 18 a 24 anos de idade corresponde a $17,6 \%$ da média de desocupação total da população brasileira; percentual que se eleva para 20,6\%, quando se considera os jovens nordestinos, denotando claro desequilíbrio regional nas oportunidades (INSTITUTO..., 2010).

Ressalta-se que a situação dos jovens brasileiros se agrava na Região Nordeste, que tem um dos menores rendimentos do Brasil e é a região com a maior população em situação de extrema pobreza do país. $\mathrm{O}$ presente estudo, pretende investigar o burnout no contexto de trabalho de jovens aprendizes que realizam atividades laborais em Recife-PE. Justifica-se a pesquisa nesse local por meio dos aspectos econômicos explicitados, e considerando a situação socioeconômica de jovens nordestinos. Jovens inseridos em realidades socioeconômicas precárias, são mais tendenciosos a percorrerem trajetórias de vida consideradas desestruturantes e adentram no mercado de trabalho por necessidade, não por escolha (GUERREIRO; ABRANTES, 2005).

Paiva et al. (2013), Tucker e Loughlin (2006) e Difiori et al. (2014), afirmam que jovens trabalhadores são mais suscetíveis a situaçôes de pressão ou estresse no trabalho. Os referidos autores explicitam que isso se dá devido aos jovens, geralmente, não terem experiência no mercado de trabalho e não desenvolverem papéis de agentes em suas atividades, sendo assim, frequentemente vítimas de circunstâncias opressoras e violentas no trabalho. Constanzi (2009) e Paiva (2014) apontam ainda, que, normalmente, os jovens entram no mercado de trabalho por meio de trabalhos informais, com baixos salários e em atividades consideradas de baixo status social, mesmo com formação educacional elevada.

Bendassolli e Soboll (2011), por sua vez, consideram que os jovens podem ser caracterizados dentro das populaçôes mais suscetíveis a vivenciarem estresse crônico ou doenças como burnout, que pertencem ao grupo de patologias nexo-causais com o trabalho (patologias relacionadas à própria atividade; 
patologias associadas à solidão ou ao sentimento de indeterminação no trabalho; patologias associadas aos maus-tratos e à violência no trabalho ou patologias de performance, consideradas como consequências dos possíveis conflitos provenientes da entrada de sujeitos sem experiência no mercado de trabalho).

A relevância social dessa pesquisa reside na compreensáo do comportamento e dos sentimentos desse grupo de trabalhadores, sendo uma maneira de refletir sobre os riscos que estáo suscetíveis esses jovens e a necessidade de prevenção a patologias do trabalho. Destaque-se, também, a baixa frequência de artigos acadêmicos no país, que buscam investigar questốes voltadas especificamente às idiossincrasias da juventude e sua relaçáo com o adoecimento mental no trabalho. Há, contudo, exceções, com ênfase à literatura internacional, que consideram os jovens como uma população com características particulares no mundo do trabalho, participando na maioria das vezes, de atividades laborais precárias (PAIVA et al., 2013; BARLING; KELLOWAY; FRONE, 2005; KELLOWAY; BARLING; HURRELL, 2006; LOUGHLIN; LANG, 2005; OLIVEIRA, 2011; PAIVA, 2012; PERRY; PARLAMIS, 2006; POCHMANN, 1998; TUCKER; LOUGHLIN, 2006; AMORIM, 2011; CONSTANZI, 2009; VENCO, 2009).

Com relação à terapia ocupacional e à atuação desses profissionais junto à saúde do trabalhador, Lancman e Ghirardi (2002) explicitam que os terapeutas ocupacionais atuam no tratamento de trabalhadores acometidos por doenças físicas e psíquicas. Nesse direcionamento, Nascimento e Souza (2018) explicitam que a saúde do trabalhador é um tema interdisciplinar, e segundo a resoluçáo no 459 , de 20 de novembro de 2015 do Conselho Federal de Fisioterapia e Terapia Ocupacional (COFFITO), é atribuição do terapeuta ocupacional, no âmbito do trabalho, promover açóes profissionais de alcance individual e/ou coletivo; de promoção à saúde; de prevenção da incapacidade temporária/permanente, progressiva/regressiva, estável/ intermitente ou contínua para o trabalho; e de reabilitação no âmbito da terapia ocupacional (BRASIL, 2015).

O burnout é considerado como uma patologia do trabalho pela Classificação Internacional de Doenças (CID-10) (BRASIL, 2016). Alguns dos seus principais sintomas são: fadiga, distúrbio do sono, dores musculares, cefaleia, irritabilidade, agressividade, incapacidade de relaxar, falta de atenção, alteraçôes de memória, lentidão, isolamento, onipotência, perda de interesse pelo trabalho ou lazer, ironia, absenteísmo, entre outros. O prognóstico está ligado a características pessoais e de trabalho como idade, sexo, nível educacional, tipo de ocupação, tempo, instituição, turno, relacionamento e assédio (CARVALHO; MAGALHÃES, 2011).

O estresse ocupacional também é frequentemente associado a um trabalho desgastante, física e emocionalmente. Porém, apesar de ser confundido muitas vezes com a síndrome de burnout, aquele se distingue desta, em função de processos ligados a eventos de tensão ou fontes de pressão diárias. A literatura indica que o estresse pode predispor a presença do burnout, bem como influenciar no desenvolvimento da síndrome (ROAZZI; CARVALHO; GUIMARĀES, 2000; ABREU et al., 2002).

Para compreender uma possível incidência da síndrome de burnout entre jovens trabalhadores, utilizou-se nesta pesquisa o modelo teórico de Maslach sobre burnout, analisado por meio da aplicação do Inventário de Maslach sobre Burnout (MBI), em função de sua grande difusão no meio acadêmico. O modelo apresenta três dimensôes: a exaustão emocional (sentimentos de esgotamento emocional no trabalho), a existência de despersonalizaçáo ou cinismo (relacionamentos negativos com as pessoas no trabalho) e a falta de realizaçáo no trabalho (sensaçóes de ineficácia e frustração na atividade laboral).

Cada dimensão do modelo pode ser analisada separadamente. Médias maiores ou menores nas dimensóes indicam maior ou menor incidência ou possibilidade de desenvolver burnout. Alguns estudos internacionais com jovens trabalhadores já indicam que essa população se encontra a caminho do desenvolvimento da síndrome, mesmo com menos tempo de trabalho que outras faixas etárias (REINARDY, 2011; GOULD et al., 2013; DIFIORI et al., 2014). Ainda, Souza, Helal e Paiva (2017) entrevistaram jovens aprendizes e perceberam através de seus relatos a vivência de situações propícias ao desenvolvimento da síndrome.

Com vistas a contribuir com esse debate, o objetivo geral deste estudo é investigar o burnout, analisando como estão configuradas a realização profissional, exaustão emocional e despersonalização no trabalho de jovens trabalhadores.

\section{Metodologia}

A pesquisa pode ser caracterizada como descritiva, de campo, e com abordagem quantitativa. O lócus de pesquisa foi o Ensino Social Profissionalizante (ESPRO) de Recife - PE, uma organização sem fins lucrativos com objetivo de capacitar profissionalmente jovens para o mercado de trabalho. A instituiçáo foi fundada em 1979, baseada na responsabilidade e inserção social, tendo já encaminhado mais de 20 mil 
jovens por ano e em situação de vulnerabilidade social para o mercado de trabalho. A ESPRO tem sede em São Paulo e filiais localizadas na cidade do Rio de Janeiro, Belo Horizonte, Curitiba, Recife, Brasília, Campinas e Porto Alegre. A escolha desta organização para a pesquisa se justifica devido à sua importância em nível nacional e regional, sua relevância social devido ao objetivo do seu trabalho, e ao critério de intencionalidade.

Cumpre informar que este estudo é parte de um projeto maior, aprovado pelo Conselho Nacional de Desenvolvimento Científico e Tecnológico (CNPq) no Edital Chamada Universal MCTI/CNPq no 14/2014. O estudo maior teve como objetivo principal "analisar como se encontram configurados determinados comportamentos e atitudes de jovens trabalhadores, na percepção deles próprios" e nele foram analisados fenômenos como: valores organizacionais, valores no trabalho, vínculos organizacionais, comprometimento organizacional, justiça organizacional, atitudes retaliatórias, qualidade de vida no trabalho, estresse ocupacional, burnout e percepçôes temporais. A pesquisa completa foi realizada em três ESPRO do Brasil: a de Belo Horizonte, Salvador e a de Recife. A coleta de dados continha questionários padronizados e validados referentes a mensuração de cada fenômeno indicado. Todos os jovens respondentes estavam matriculados na ESPRO das cidades brasileiras indicadas e responderam a todos os questionários, inclusive ao questionário sobre burnout, analisado nesse artigo.

Devido à grande quantidade de resultados obtidos nas coletas de dados do projeto maior, optou-se por analisar cada fenômeno separadamente. Foram elaborados artigos, monografias, dissertaçóes e teses, em diversos lugares do país onde foram realizadas as coletas, focalizando os diversos fenômenos analisados nas diversas ESPRO do Brasil. Esse artigo representa um recorte desse projeto maior, explicitando as reflexôes especificamente provenientes das análises sobre a mensuração do fenômeno burnout com jovens da ESPRO Recife. O projeto completo foi submetido e aprovado pelo Comitê de Ética em Pesquisa (CEP) da Universidade Federal de Minas Gerais (UFMG), com pareceres de permissão com números 710.503 e 240.802 .

Com relação à aplicação do questionário e assinatura dos documentos éticos, algumas etapas foram seguidas. Em primeiro lugar, os pesquisadores entraram nas salas de aula da ESPRO e apresentaram o projeto, seus objetivos e a importância de que os jovens participassem. Foi esclarecido que a participação era voluntária e que os participantes poderiam desistir a qualquer momento. Todos os jovens que receberam o questionário aceitaram respondê-lo voluntariamente, e eram maiores de 18 anos. Os jovens assinaram as duas vias do Termo de consentimento livre e esclarecido (TCLE) no modelo prescrito pelo Comitê de Ética em Pesquisa da UFMG. Os TCLE foram entregues aos pesquisadores pelos jovens, junto com os questionários respondidos.

Com relação à amostra, os jovens foram selecionados por meio do critério de acessibilidade e de acordo com sua disponibilidade em participar do estudo, ou seja, responderam os questionários todos os jovens maiores de 18 anos, que estavam nas dependências do Ensino Social Profissionalizante (ESPRO) da cidade de Recife - PE, nos dias 30 e 31 de março e $1^{\circ}$ de abril do ano de 2016, totalizando 239 respondentes.

$\mathrm{O}$ critério de inclusão na amostra foi o jovem estar trabalhando, ser aluno da ESPRO Recife, ter mais de 18 anos e estar presente no dia da coleta de dados.

$\mathrm{O}$ instrumento continha um questionário de identificação do participante, já utilizado em outras pesquisas brasileiras com o mesmo tema e população (PAIVA, 2014; SOUZA; HELAL; PAIVA, 2017). Esse questionário continha uma parte referente aos dados sociodemográficos (sexo, idade, escolaridade, estado civil, etnia, escolaridade do pai e mãe) e de dados profissionais (ramo do trabalho, tempo que trabalha, cargo, renda...).

Para mensuraçáo quantitativa do burnout, foi utilizado o inventário de Maslach sobre burnout (MBI), elaborado pela psicóloga Cristina Maslach (2003). Justifica-se seu uso por este ser um instrumento validado nacional e internacionalmente e amplamente utilizado por pesquisadores que estudam o tema. Schaufeli e Greenglass (2001) consideram que o modelo teórico de Maslach sobre burnout - através do qual foi desenvolvido o MBI - é o modelo mais utilizado para medir o burnout, presente em $90 \%$ das pesquisas científicas que averiguam o tema. O questionário é uma escala likert de 22 variáveis referentes às três dimensóes previstas no modelo teórico de burnout (MASLACH, 2003), a saber:

Cumpre esclarecer que a exaustão emocional, no $\mathrm{MBI}$, possui nove itens dedicados a sua mensuração, a saber: sinto-me emocionalmente decepcionado com meu trabalho; quando termino minha jornada de trabalho, sinto-me esgotado; quando me levanto pela manhã e enfrento outra jornada de trabalho, sinto-me fatigado; sinto que trabalhar todo o dia com pessoas me cansa; sinto que meu trabalho está me desgastando; sinto-me frustrado com meu trabalho; sinto que estou trabalhando demais; sinto que trabalhar em contato direto com as pessoas me estressa; sinto como se estivesse no limite de minhas 
possibilidades. A despersonalização, cinco itens: sinto que estou tratando algumas pessoas de meu trabalho como se fossem objetos impessoais; sinto que me tornei mais duro com as pessoas desde que comecei esse trabalho; preocupo-me se esse trabalho está me enrijecendo emocionalmente; sinto que realmente não me importa o que ocorra com as pessoas as quais tenho que atender profissionalmente; parece-me que os receptores de meu trabalho culpam-me por alguns de seus problemas; e, por último, a diminuição da realização pessoal no trabalho, oito itens no inventário: sinto que posso entender facilmente as pessoas que tenho que atender; sinto que trato com muita eficiência os problemas das pessoas as quais tenho que atender; sinto que estou exercendo influência positiva na vida das pessoas que tenho que atender; sinto-me vigoroso em meu trabalho; sinto que posso criar um clima agradável em meu trabalho; sinto-me estimulado depois de haver trabalhado diretamente com quem tenho que atender; creio que consigo coisas valiosas nesse trabalho; no meu trabalho, eu manejo os problemas emocionais com muita calma (CARLOTTO; PALAZZO, 2006, p. 1019).

As respostas às perguntas indicadas no inventário devem ser somadas e divididas de forma a obter a média aritmética das pontuaçôes obtidas em cada dimensáo, uma vez que, tanto no modelo quanto no instrumento, as variáveis referentes às três dimensôes do burnout são consideradas independentes e podem ser mensuradas e analisadas separadamente. Por ser um questionário validado nacional e internacionalmente, com amplo uso dos pesquisadores sobre o tema, o $\mathrm{MBI}$, teoricamente, tem validez fatorial e consistência interna.

Os dados coletados através dos dois instrumentos utilizados neste trabalho foram organizados em planilhas dos softwares Excel e SPSS, com o objetivo de averiguar possíveis erros nas respostas ou falhas que pudessem prejudicar análises estatísticas posteriores. Por isso, os respondentes que apresentavam missing values em variáveis categóricas foram excluídos, porque se acredita que não convém substituir características do perfil pessoal dos respondentes por respostas baseadas em análises meramente frequenciais. Da mesma forma, as respostas ao MBI de tais respondentes foram excluídas para equiparar a amostra e favorecer as análises estatísticas. Após essa análise preliminar de dados, dos 239 respondentes, 213 permaneceram na pesquisa.

Após a fase preliminar de tabulação dos dados nos softwares, a análise dos dados foi dividida em quatro etapas, a saber: análise descritiva da amostra (com os dados provenientes do questionário de identificação), análise de mensuração das variáveis (ou análise fatorial) do MBI, análise através de ANOVA e análises ou testes adicionais.

Foram feitas análises de frequência e percentual das principais variáveis consideradas no questionário de identificaçáo do entrevistado (ou questionário de dados sociodemográficos e de trabalho), com o fim de mostrar o panorama geral do perfil dos entrevistados ou a descrição da amostra.

Em relaçáo ao inventário sobre a síndrome de burnout validado por Maslach (MBI), procedeu-se a uma análise fatorial, com o objetivo de compreender o quanto cada pergunta responde às necessidades do contexto da pesquisa e, principalmente, se se enquadram nos três componentes principais que se referem à teoria de burnout utilizada (Realizaçáo profissional, Exaustão emocional e Despersonalização/Cinismo). Assim, foi realizado o método de extração de fatores por meio de componentes principais com rotação ortogonal Varimax.

A partir da obtenção dos resultados do coeficiente alfa de Cronbach $(\langle=0,882)$, percebeu-se a adequação do questionário. Foram assim, para cada construto, confirmados, além de uma confiabilidade adequada e fatores satisfatórios nos testes de KMO e Barlett, a existência de componentes únicos em cada construto - que se referem às dimensóes da Realizaçáo Profissional (RP) Exaustáo Emocional (EM) e Despersonalização (DES), permitindo que no contexto dessa pesquisa, as variáveis sejam consideradas em seus componentes originais, explicitados e elaborados a partir de Maslach e Jackson (1981) e de Maslach, Schaufeli e Leiter (2001).

\section{Resultados}

Com relação ao perfil geral dos 213 respondentes que continuaram na pesquisa após a análise preliminar têm-se: predominância de indivíduos do gênero feminino (54,9\%), com o ensino médio completo $(46,5 \%)$, e a maioria dos pais $(46 \%)$ e das mães (39\%) dos entrevistados cursou o ensino médio completo. O tempo de trabalho da maioria da população foi de seis meses a um ano (72,3\%), e o ramo de trabalho mais frequente foi o da indústria (44,1\%). Observou-se ainda que, 32,9\% dos respondentes possui renda familiar entre um e dois salários mínimos, sendo este o valor mais baixo estabelecido questionário de identificação aplicado.

A análise estatística descritiva dos resultados do MBI foi dividida em três níveis de avaliação: elevado (acima de 3,9); mediano (entre 3,0 e 3,9) e baixo (abaixo de 2,9), da mesma forma que tratou do assunto um estudo anterior brasileiro (PAIVA et al., 2013) com a mesma população desta 
pesquisa. As médias por dimensão e o nível geral das dimensóes se encontram na Tabela 1, a seguir:

Com o objetivo de comparar dois, três ou mais grupos de respondentes, procedeu-se à análise de médias por meio do Teste-T de amostras independentes e do método ANOVA, a depender da quantidade de grupos analisados (2 ou mais). As variáveis submetidas aos testes foram: gênero (GEN), idade (IDADE), escolaridade do jovem (ESCOLARIDADE), escolaridade do pai (ESCOL_PAI), escolaridade da mãe (ESCOL_MÃE), tempo de trabalho (TEMPO_TRAB), ramo do trabalho (RAMO_TRAB), renda da casa (RENDA_CASA), recebe ou não salário (RECEBE_SAL). Tais variáveis foram escolhidas tendo em vista tanto os interesses de investigação dessa pesquisa, como levando em consideração os estudos mais acessados e citados sobre o tema, que analisam em sua maioria a idade e o gênero dos participantes.

$\mathrm{Na}$ análise, foram levados em consideração o P-valor (ou Sig.), o df e o Z, de cada variável, relacionados a cada componente ou dimensáo do burnout. Sendo assim, foram construídas 3 tabelas, referentes a cada componente ou dimensão (Realização Profissional, Exaustão Emocional, Despersonalização).

Com relação à Realização Profissional, são apontadas na Tabela 2 diferenças marginais entre grupos nas variáveis "Escolaridade da mãe", e "tempo

Tabela 1. Dimensões e níveis gerais.

\begin{tabular}{|c|c|c|c|c|c|}
\hline Dimensões & Mínimo & Máximo & Var. & Médias & $\begin{array}{l}\text { Nível geral } \\
\text { da dimensão } \\
\text { pela média }\end{array}$ \\
\hline Realização profissional & 1,3 & 5,9 & 6 & 3,9 & MEDIANO \\
\hline Exaustão emocional & 1 & 5 & 1,0 & 2,6 & BAIXO \\
\hline Despersonalização/Cinismo & 1 & 5 & 1,9 & 2,3 & BAIXO \\
\hline GERAL & 1,1 & 5,3 & 1,8 & 2,9 & BAIXO \\
\hline
\end{tabular}

Tabela 2. Resultados sobre Realização Profissional.

\begin{tabular}{|c|c|c|c|c|}
\hline \multicolumn{5}{|c|}{ REALIZAÇÃO PROFISSIONAL } \\
\hline Variável categórica & ANOVA/TESTE T & $\begin{array}{c}\text { Sig. } \\
\text { (P-valor). }\end{array}$ & Df & \\
\hline \multirow[t]{3}{*}{ Idade } & Entre os grupos & 966 & 7 & ,267 \\
\hline & Nos grupos & & 205 & \\
\hline & Total & & 212 & \\
\hline \multirow[t]{3}{*}{ Gênero } & Variâncias iguais assumidas & 142 & 211 & 2,173 \\
\hline & & & 189,4 & \\
\hline & Variâncias iguais não assumidas & & 06 & \\
\hline \multirow[t]{3}{*}{ Escolaridade } & Entre os grupos & ,278 & 4 & 1,284 \\
\hline & Nos grupos & & 208 & \\
\hline & Total & & 212 & \\
\hline \multirow[t]{3}{*}{ Escolaridade do pai } & Entre os grupos & 165 & 6 & 1,544 \\
\hline & Nos grupos & & 206 & \\
\hline & Total & & 212 & \\
\hline \multirow[t]{3}{*}{ Escolaridade da mãe } & Entre os grupos & 047 & 6 & 2,170 \\
\hline & Nos grupos & & 206 & \\
\hline & Total & & 212 & \\
\hline \multirow[t]{3}{*}{ Renda } & Entre os grupos & 243 & 8 & 1,305 \\
\hline & Nos grupos & & 204 & \\
\hline & Total & & 212 & \\
\hline \multirow[t]{3}{*}{ Tempo de trabalho } & Entre os grupos &, 048 & 6 & 2,163 \\
\hline & Nos grupos & & 206 & \\
\hline & Total & & 212 & \\
\hline \multirow{3}{*}{ Ramo de trabalho } & Entre os grupos & 286 & 8 & 1,224 \\
\hline & Nos grupos & & 204 & \\
\hline & Total & & 212 & \\
\hline \multirow[t]{2}{*}{ Recebe salário } & Variâncias iguais assumidas & ,760 & 211 & 0,93 \\
\hline & Variâncias iguais não assumidas & & 16,101 & \\
\hline
\end{tabular}

Sig = nível de significância; $\mathrm{T}$ = teste de hipótese "'Student"; $\mathrm{Df}=$ graus de Liberdade. 
de trabalho". Com relação ao tempo de trabalho, mesmo com diferenças marginais apontadas pelo teste ANOVA na comparação entre grupos, não foi possível encontrar a diferença a partir dos testes Post-Hoc de Barley ou de Tuckey, dificultando a identificação da diferença das médias, o que pode apontar que o $\mathrm{P}$-valor um pouco inferior ao limite de confirmaçáa da hipótese nula $(0,05)$, pode ter sido dado devido a dispersão de dados pelo grande número de grupos indicados no questionário para essa variável categórica (9 grupos), ou ao acaso. Já na variável escolaridade da mãe, foram encontradas diferenças significativas entre indivíduos que possuem o ensino fundamental completo e o ensino superior completo (Tabela 2).
As médias da realização profissional para o ensino fundamental completo foram maiores do que para o ensino superior completo, como indicado na Tabela 3 a seguir. Isso demonstra que jovens aprendizes com mães detentoras de ensino fundamental completo são mais realizados profissionalmente do que os com mães que possuem o ensino superior completo.

Nos resultados acerca do componente Exaustão Emocional, percebe-se a diferença apontada entre médias na variável Gênero. Por meio do Teste-T, na comparação entre essa dimensão, e os gêneros dos jovens respondentes no presente contexto de pesquisa, constatou-se ligeira diferença entre as médias, que podem ser constatadas na Tabela 4 a seguir.

Tabela 3. Realização Profissional/Escolaridade da mãe (diferenças das médias entre grupos).

\begin{tabular}{c|c|ccc|c}
\hline \multicolumn{3}{c}{ Estatísticas de grupo } \\
\hline & $\begin{array}{c}\text { Escolaridade da mãe } \\
\text { (ESCOL_MÃE) }\end{array}$ & $\mathbf{N}$ & Média & $\begin{array}{c}\text { Desvio } \\
\text { Padrão }\end{array}$ & $\begin{array}{c}\text { Erro } \\
\text { padrão da } \\
\text { média }\end{array}$ \\
\hline $\begin{array}{c}\text { Realização profissional } \\
\text { (RP_geral) }\end{array}$ & $\begin{array}{c}\text { Ensino fundamental completo } \\
\text { Ensino superior completo }\end{array}$ & 23 & 4,2565 &, 70054 &, 14607 \\
\hline
\end{tabular}

Tabela 4. Resultados sobre Exaustão Emocional.

\begin{tabular}{|c|c|c|c|c|}
\hline \multicolumn{5}{|c|}{ EXAUSTÃO EMOCIONAL } \\
\hline Variável categórica & ANOVA/TESTE T & Sig. (P-valor). & Df & $\mathrm{Z}$ \\
\hline \multirow[t]{3}{*}{ Idade } & Entre os grupos & 966 & 7 & 267 \\
\hline & Nos grupos & & 205 & \\
\hline & Total & & 212 & \\
\hline \multirow[t]{3}{*}{ Gênero } & Variâncias iguais assumidas & 040 & 211 & 4,263 \\
\hline & & & 209,6 & \\
\hline & Variâncias iguais não assumidas & & 51 & \\
\hline \multirow[t]{3}{*}{ Escolaridade } & Entre os grupos & ,230 & 4 & 1,415 \\
\hline & Nos grupos & & 208 & \\
\hline & Total & & 212 & \\
\hline \multirow{3}{*}{ Escolaridade do pai } & Entre os grupos & ,375 & 6 & 1,081 \\
\hline & Nos grupos & & 206 & \\
\hline & Total & & 212 & \\
\hline \multirow{3}{*}{ Escolaridade da mãe } & Entre os grupos &, 519 & 6 & 869 \\
\hline & Nos grupos & & 206 & \\
\hline & Total & & 212 & \\
\hline \multirow[t]{3}{*}{ Renda } & Entre os grupos & 642 & 8 & ,755 \\
\hline & Nos grupos & & 204 & \\
\hline & Total & & 212 & \\
\hline \multirow[t]{4}{*}{ Tempo de trabalho } & Entre os grupos & ,536 & 6 & 845 \\
\hline & & & 206 & \\
\hline & Nos grupos & & 212 & \\
\hline & Total & & & \\
\hline \multirow[t]{3}{*}{ Ramo de trabalho } & Entre os grupos & ,431 & 8 & 1,008 \\
\hline & Nos grupos & & 204 & \\
\hline & Total & & 212 & \\
\hline \multirow[t]{2}{*}{ Recebe salário } & Variâncias iguais assumidas & ,265 & 211 & 1,249 \\
\hline & Variâncias iguais não assumidas & & 17,020 & \\
\hline
\end{tabular}

$\mathrm{Z}=$ número de desvios padrão acima ou abaixo da média da população; $\mathrm{Df}=$ graus de Liberdade. 
Tabela 5. Resultados sobre despersonalização.

\begin{tabular}{|c|c|c|c|c|}
\hline \multicolumn{5}{|c|}{ DESPERSONALIZAÇÃO } \\
\hline Variável categórica & ANOVA/TESTE T & Sig. (P-valor). & Df & $\mathrm{Z}$ \\
\hline \multirow[t]{3}{*}{ Idade } & Entre os grupos & 613 & 7 & 613 \\
\hline & & & 205 & \\
\hline & Nos grupos & & 212 & \\
\hline \multirow[t]{3}{*}{ Gênero } & Variâncias iguais assumidas & 685 & 211 & 165 \\
\hline & & & 198,9 & \\
\hline & Variâncias iguais não assumidas & & 85 & \\
\hline \multirow[t]{3}{*}{ Escolaridade } & Entre os grupos & 0,52 & 4 & 2,393 \\
\hline & Nos grupos & & 208 & \\
\hline & Total & & 212 & \\
\hline \multirow[t]{3}{*}{ Escolaridade do pai } & Entre os grupos & 478 & 6 & 925 \\
\hline & Nos grupos & & 206 & \\
\hline & Total & & 212 & \\
\hline \multirow[t]{3}{*}{ Escolaridade da mãe } & Entre os grupos & ,882 & 6 &, 395 \\
\hline & Nos grupos & & 206 & \\
\hline & Total & & 212 & \\
\hline \multirow[t]{4}{*}{ Renda } & Entre os grupos & ,227 & 8 & 1,336 \\
\hline & & & 204 & \\
\hline & Nos grupos & & 212 & \\
\hline & Total & & & \\
\hline \multirow[t]{3}{*}{ Tempo de trabalho } & Entre os grupos & ,641 & 6 &, 712 \\
\hline & Nos grupos & & 206 & \\
\hline & Total & & 212 & \\
\hline \multirow[t]{3}{*}{ Ramo de trabalho } & Entre os grupos & 286 & 8 & 1,224 \\
\hline & Nos grupos & & 204 & \\
\hline & Total & & 212 & \\
\hline \multirow[t]{2}{*}{ Recebe salário } & Variâncias iguais assumidas & ,760 & 211 & 0,93 \\
\hline & Variâncias iguais não assumidas & & 16,101 & \\
\hline
\end{tabular}

$\mathrm{Z}=$ número de desvios padrão acima ou abaixo da média da população; $\mathrm{Df}=$ graus de Liberdade.

De acordo com a análise, percebeu-se que os respondentes jovens do gênero masculino $(m=2,76)$ são ligeiramente mais exauridos emocionalmente do que as jovens mulheres $(\mathrm{m}=2,48)$.

O último componente, que se refere aos aspectos sobre Despersonalização/cinismo, não apresentou diferenças entre as médias dos grupos analisados, em nenhuma variável categórica testada, como consta na Tabela 5.

No que tange às variáveis categóricas analisadas junto à dimensão da despersonalização, percebe-se que nenhuma delas apresentou P-valor menor do que 0,05 , indicando que os grupos analisados não se diferenciam estatisticamente.

\section{Discussão}

Sobre os resultados da Tabela 1, mesmo que nesta pesquisa não se tenha a intençáo de diagnosticar burnout - e que os resultados do inventário náo apontem que a maioria dos jovens consideram que vivenciam situaçóes que podem acarretar em burnout -, compreendemos que as organizações e os pesquisadores envolvidos com estudos sobre organização do trabalho, psicopatologia e saúde mental, devem averiguar mais profundamente o discurso desses trabalhadores. O fato de um ou mais jovens terem respondido valores máximos em algumas das dimensôes, chama atenção para a necessidade de realizar novas abordagens de pesquisa para coletar dados mais específicos sobre essa situação de trabalho, e práticas que fomentem um maior bem-estar e qualidade de vida desses jovens trabalhadores.

Os resultados apresentados na Tabela 1 indicam que os valores máximos encontrados são ilustrativos de uma avaliação elevada ou muito elevada (de 5 a 5,9) de dimensões de burnout. Assim, por mais que as médias gerais da amostra pareçam não convergir para uma média alta nos componentes do $\mathrm{MBI}$, alguns respondentes apresentaram indícios significativos de desenvolvimento da síndrome, indicando ser algo presente entre os jovens. 
Os resultados indicaram que jovens que possuem pais com maior escolaridade se sentem menos realizados. Tal resultado pode estar associado às expectativas elevadas que os pais porventura tenham em relação a seus filhos, podendo contribuir para a sensação de menor realizaçáo por parte dos jovens. A escassez na literatura sobre estudos que associam burnout e escolaridade dos pais limita as reflexôes acerca desse achado.

Contudo, ao identificar menores níveis de realização em jovens que têm pais com maiores níveis de escolarização, reflete-se sobre a necessidade do desenvolvimento de postos de trabalho no mercado, que atendam a necessidades pessoais e motivacionais de cada trabalhador. Os jovens, mesmo com pais mais qualificados podem, por necessidade, acabar optando por postos de trabalho que não correspondem às qualificaçóes de sua família ou perspectivas futuras de carreira, porque necessitam, primeiramente, se inserir no mercado de trabalho.

O fato mencionado anteriormente foi explicitado em um estudo de Letelier (1999), que aponta a falta de investimento da sociedade na geraçáo de novos postos de trabalho e menciona que o investimento demasiado em educaçáo, tem como consequência a subutilizaçáo da máo de obra, responsável por possíveis frustraçóes profissionais. Sendo assim, é possível que jovens que vivenciam realidades de famílias com níveis de qualificação mais altos, vivenciem maior frustração e consequente falta de realização profissional ao não conseguirem as oportunidades de trabalho almejadas. Souza, Helal e Paiva (2017) identificaram que muitos jovens aprendizes exercem esses cargos pela remuneraçẫo e vontade de sustentar cursos particulares em outras áreas, e não pelo desejo pessoal pelo cargo. Em seu estudo, Letelier (1999) aponta ainda que a falta de investimento em geração de novos postos de trabalho gera "[...] frustração das aspiraçóes que a população vem depositando na elevação dos níveis educacionais" (LETELIER, 1999, p. 137).

Alguns dos estudos mais acessados sobre o tema como os de Gil-Monte (2002), Grau, Suñer e García (2005) e Grau et al. (2009), apontam que gênero e exaustáo emocional não possuem quaisquer relaçóes. Estudos internacionais de periódicos científicos indexados, com estudantes (O'DONOGHUE; NEIL, 2015) e com enfermeiras (QUEIROS et al., 2013) também não encontram diferenças estatísticas significativas entre gênero, burnout e exaustão emocional.

Mais uma vez, assim como Paiva e colaboradores (2013) apontaram em seu estudo, sugere-se a existência de características específicas e paradigmáticas do público jovem, como o da amostra desta pesquisa. Diante disso, são necessários novos estudos específicos e mais aprofundados sobre essa população, bem como o desenvolvimento de novos questionários e outros métodos de pesquisa que busquem explicar, por exemplo, a maior exaustão emocional entre jovens homens do que entre mulheres.

No último componente, que se refere aos aspectos sobre Despersonalização/cinismo, náo foram encontradas diferenças estatísticas entre médias ou grupos. Ainda, não foram identificados na literatura artigos que indiquem resultados contrários aos desta pesquisa, considerando esta dimensão. Outros estudos também não encontraram relaçóes entre os grupos de jovens e a despersonalização (PAIVA et al., 2013; SOUZA; HELAL; PAIVA, 2017). Sugere-se que tal questão seja melhor explorada em outras pesquisas sobre o tema.

\section{Conclusão}

A análise das respostas do MBI indica, em linhas gerais, que os jovens entrevistados náo estão vivenciando burnout de modo elevado. Porém, cumpre indicar que há respondentes que apresentaram altos valores em alguns dos três componentes (constataçáo dada a partir da observação dos valores máximos em cada componente), o que pode indicar comportamento tendencioso a estresse crônico, e possibilidade de futuro desenvolvimento de sintomas típicos do burnout - mesmo que na minoria da amostra.

Ainda, percebe-se que os jovens apresentam altos níveis de realizaçáo profissional, o que parece incentivar para que tenham índices de baixo a mediano também nas dimensóes da exaustão emocional e despersonalização. Sobre a realização profissional, foi constatada a diferença de médias entre jovens que são filhos de mães com ensino fundamental completo e de mães com ensino superior completo, indicando que jovens com pais com maior realização profissional têm pais com menor escolaridade. Apesar de escassez de evidências que auxilie a discutir esse ponto, a literatura coloca que o aumento de oportunidades educacionais, tanto dos jovens quanto da sua família, não está vinculado ao aumento de postos de trabalho dignos e adequados aos desejos e necessidades pessoais dos trabalhadores, o que pode gerar frustração.

É necessário, também, compreender a realização profissional de maneira mais aprofundada, por meio de pesquisas que busquem compreender os relatos dos jovens e identificar se a realizaçáo profissional está ligada à remuneração, ou ao desejo pessoal de permanecer nesse trabalho. 
Sobre os resultados dos testes adicionais para o componente da exaustão emocional, são indicadas diferenças entre gêneros, demonstrando que jovens homens se percebem mais exauridos emocionalmente com o desenvolvimento do seu trabalho do que as mulheres.

Com relação à agenda de pesquisas, sugere-se atenção ao médio e alto nível escolar dos pais e das mães dos jovens respondentes. Tais dados podem ser de interesse de outras pesquisas que tenham o objetivo de compreender motivos e implicações de um alto nível de escolaridade associado a rendas familiares muito abaixo da média - 1 a 2 salários mínimos.

Ainda, de acordo com o alto índice de jovens aprendizes respondentes que trabalham no ramo da indústria, sugere-se que tal setor, em especial, comece a observar os aspectos subjetivos ligados ao trabalho dessas pessoas. Acredita-se que, ao considerar esses elementos, o setor tenderia a observar resultados melhores tanto na satisfação pessoal dos jovens, quanto no desempenho positivo desse trabalhador, o que, certamente, traria influência no desempenho das empresas.

As limitações encontradas nesta pesquisa referem-se à quantidade de questionários que foram excluídos da análise da pesquisa devido à falta de muitas respostas dos entrevistados (missing values). É possível que esse fato tenha sido dado devido à extensão do questionário respondido pelos participantes.

Acredita-se, por fim, que este artigo pode também contribuir com as organizaçóes e com o Estado, porque, ao compreender as características de trabalho especiais desses jovens, poderão ser empreendidas açóes de prevenção de fenômenos do adoecimento em longo prazo, assim como políticas públicas que reflitam sobre a geração de postos de trabalho, a inserçáo e a permanência de jovens no mercado de trabalho.

Concluímos que a existência de jovens que apresentam sintomas de estresse crônico, ou com indícios de um possível desenvolvimento do burnout leva a necessidade de maior reflexão sobre a individualidade do trabalhador, e sobre os fatores subjetivos que influenciam diretamente em participaçôes positivas nas atividades da empresa. A realização de outras pesquisas com métodos que considerem os relatos verbais de jovens inseridos no mercado de trabalho sobre satisfação com sua função, os motivos de sua inserção no mercado de trabalho e suas possíveis expectativas ou perspectivas para o futuro é necessária e esperada.

\section{Referências}

ABREU, K. L. et al. Estresse ocupacional e síndrome de burnout no exercício profissional da Psicologia. Psicologia: Ciência e Profissão, Brasília, v. 22, n. 2, p. 22-29, 2002.

AMORIM, L. A. Yum! Tem seu próprio Silvio Santos para fast food. Revista Exame, São Paulo, v. 970, p. 1-3, 2011.

BARLING, J.; KELLOWAY, E. K.; FRONE, M. R. Editor's overviews: special populations. In: BARLING, J.; KELLOWAY, E. K.; FRONE, M. R. (Org.). Handbook of work stress. Thousand Oaks: Sage, 2005. p. 401-404.

BENDASSOLLI, P. F.; SOBOLL, L. A. P. Introdução às clínicas do trabalho: aportes teóricos, pressupostos e aplicaçôes. In: BENDASSOLLI, P. F.; SOBOLL, L. A. P. (Org.). Clínicas do trabalho. São Paulo: Atlas, 2011. p. 3-21.

BRASIL. Resolução no 459, de 20 de novembro de 2015. Dispóe sobre as competências do terapeuta ocupacional na Saúde do Trabalhador, atuando em programas de estratégias inclusivas, de prevenção, proteção e recuperação da saúde. Diário Oficial [da] República Federativa do Brasil, Brasília, DF, 20 nov. 2015. Disponível em: <https://www.coffito. gov.br/nsite/?p=3220 >. Acesso em: 20 out. 2017.

BRASIL. Classificação Estatística Internacional de Doenças e Problemas Relacionados à Saúde - CID-10. Brasília: DATASUS, 2016.

CARLOTTO, M. S.; PALAZZO, L. S. Síndrome de burnout e fatores associados: um estudo epidemiológico com professores. Cadernos de Saúde Pública, Rio de Janeiro, v. 22, n. 5, p. 1017-1026, 2006.

CARVALHO, C. G.; MAGALHÃES, S. R. Síndrome de burnout e suas consequências nos profissionais de enfermagem. Revista da Universidade Vale do Rio Verde, Três Coraçóes, v. 9, n. 1, p. 200-210, 2011.

CONSTANZI, R. N. Trabalho decente e juventude no Brasil. Brasília: OIT, 2009.

DIFIORI, J. P. et al. Overuse Injuries and Burnout in youth sports: a position statement from the American Medical Society for Sports Medicine. Clinical Journal of Sport Medicine, Loughborough, v. 48, n. 4, p. 287-288, 2014.

GIL-MONTE, P. R. Influencia del género sobre el proceso de desarrollo del síndrome de quemarse por el trabajo (burnout) em profesionales de enfermería. Psicologia em Estudo, Maringá, v. 7, n. 1, p. 3-10, 2002.

GOULD, D. D. et al. The relationship between burnout and coping. Psychological Services, Washington, v. 10, n. 1, p. 37-47, 2013.

GRAU, A. et al. Influencia de factores personales, profesionales y transnacionales em el síndrome de burnout en personal sanitário hispanoamericano y español. Revista Espanola de Salud Publica, Madrid, v. 83, n. 2, p. 215-230, 2009.

GRAU, A.; SUÑER, R.; GARCÍA, M. M. Desgaste professional en el personal sanitario y su relación con los factores personales y ambientales. Gaceta Sanitaria, Barcelona, v. 19, n. 6, p. 463-470, 2005. 
GUERREIRO, M. D.; ABRANTES, P. Como tornar-se adulto: processos de transição na modernidade avançada. Revista Brasileira de Ciência do Solo, Viçosa, v. 20, n. 58, p. 157-212, 2005.

INSTITUTOBRASILEIRODEGEOGRAFIAEESTATÍSTICA - IBGE. Censo demográfico. Brasília: IBGE, 2010.

KELLOWAY, E. K.; BARLING, J.; HURRELL, J. Editor's introduction to part II. In: KELLOWAY, E. K.; BARLING, J.; HURREL, J. (Org.). Handbook of workplace violence. Thousand Oaks: Sage, 2006. p. 93-94.

LANCMAN, S.; GHIRARDI, M. I. G. Pensando novas práticas em terapia ocupacional saúde do trabalhador. Revista de Terapia Ocupacional da Universidade de São Paulo, São Paulo, v. 2, n. 13, p. 44-50, 2002.

LETELIER, G. M. E. Escolaridade e inserção no mercado de trabalho. Cadernos de Pesquisa, São Paulo, n. 107, p. 133-148, 1999.

LOUGHLIN, C.; LANG, K. Young workers. In: BARLING, J.; KELLOWAY, E. K.; FRONE, M. R. (Org.). Handbook of work stress. Thousands Oaks: Sage, 2005. p. 405-430.

MASLACH, C. Job Burnout: new directions in research and intervention. Current Directions in Psychological Science, Thousand Oaks, v. 12, n. 5, p. 189-192, 2003.

MASLACH, C.; JACKSON, S. E. The measurement of experienced burnout. Journal of Occupational Behavior, New York, v. 2, n. 2, p. 99-113, 1981.

MASLACH, C.; SCHAUFELI, W. B.; LEITER, M. P. Job burnout. Annual Review of Psychology, United States, v. 52, p. 397-422, 2001

NASCIMENTO, A. S.; SOUZA, M. B. C. A. Ergonomia e postos de trabalho: análise do ambiente de trabalho de professores da Universidade Federal da Paraíba de acordo com a NR17. Revista Interinstitucional Brasileira de Terapia Ocupacional, Rio de Janeiro, v. 2, n. 3, p. 567-583, 2018.

O'DONOGHUE, P.; NEIL, R. Relative age effect on behavioural regulation, burnout potential and anxiety of sports students. European Journal of Human Movement, Alcorcón, v. 35, p. 1-11, 2015.

OLIVEIRA, S. R. Ponto de partida: a juventude e o ingresso no mercado de trabalho. In: FERRAZ, D. L. S.; OLTRAMARI, A. P.; PONCHIROLLI, O. (Org.). Gestão de pessoas e relações de trabalho. São Paulo: Atlas, 2011. p. 89-112.

PAIVA, K. C. M. Valores organizacionais e do trabalho: um estudo com jovens trabalhadores. In: ENCONTRO DA
ASSOCIAÇÃO NACIONAL DE PÓS-GRADUAÇÃO E PESQUISA EM ADMINISTRAÇÃO, 36., 2012, Rio de Janeiro. Anais... Rio de Janeiro: ANPAD, 2012. p. 1-16.

PAIVA, K. C. M. et al. Estresse ocupacional e burnout de jovens trabalhadores. In: ENCONTRO DA ANPAD, 37. 2013, Rio de Janeiro. Anais... Rio de Janeiro, 2013. p. 1-16.

PAIVA, K. C. M. Comportamento organizacional: um estudo multitemático com jovens trabalhadores. Belo Horizonte: UFMG, 2014. Proposta de Projeto de Pesquisa Científica - Edital Chamada Universal MCTI/CNPq, n. 14/2014.

PERRY, E. L.; PARLAMIS, J. D. Age and ageism in organizations. In: KONRAD, A. M.; PRASAD, P.; PRINGLE, J. K. (Org.). Handbook of work place diversity. London: Sage, 2006. p. 345-370.

POCHMANN, M. A inserção ocupacional e o emprego dos jovens. São Paulo: ABET, 1998.

QUEIROS, C. et al. Predictors of burnout among nurses: an interactionist approach. Psicothema, Oviedo, v. 25, n. 3, p. 330-335, 2013.

REINARDY, S. Newspaper journalism in crisis: burnout on the rise, eroding young journalists' career commitment. Sage Journals, Washington, v. 12, n. 1, p. 33-50, 2011.

ROAZZI, A.; CARVALHO, A. D.; GUIMARÃES, P. V. Análise da estrutura de similaridade da síndrome de burnout: validação da escala Maslach Burnout Inventory em professores. In: ENCONTRO MINEIRO DE AVALIAÇÃO PSICOLÓGICA: TEORIA E PRÁTICA; CONFERÊNCIA INTERNACIONAL DE AVALIAÇÃO PSICOLÓGICA: FORMAS E CONTEXTOS, 8., 2000, Belo Horizonte. Anais... Belo Horizonte: Centro Universitário Newton Paiva, 2000. p. 1-33.

SCHAUFELI, W. B.; GREENGLASS, E. R. Introduction to special issue on burnout and health. Psychology \& Health, Abingdon, v. 16, n. 5, p. 501-510, 2001

SOUZA, M. B. C. A.; HELAL, D. H.; PAIVA, K. C. M. Burnout e jovens trabalhadores. Cadernos Brasileiros de Terapia Ocupacial, São Carlos, v. 25, n. 4, p. 751 763, 2017.

TUCKER, S.; LOUGHLIN, C. Young workers. In: KELLOWAY, E. K.; BARLING, J.; HURRELL, J. (Org.). Handbook of workplace violence. Thousand Oaks: Sage, 2006. p. 417-444.

VENCO, S. Centrais de tele atividades. In: ANTUNES, R.; BRAGA, R. (Org.). Info proletários. São Paulo: Boitempo, 2009. p. 153-172.

\section{Contribuição dos Autores}

Marina Batista Chaves Azevedo de Souza: Concepção do projeto, análise e interpretaçáo dos dados e redação do artigo. Diogo Henrique Helal e Kely César Martins de Paiva: Revisão crítica relevante do conteúdo intelectual. Todos os autores aprovaram a versão final do texto.

\section{Fonte de Financiamento}

CNPq - Edital Chamada Universal MCTI/CNPq no 14/2014 e Coordenação de Aperfeiçoamento de Pessoal de Nível Superior - CAPES (Código 001). 